\title{
Improving Communication Sensitive Parallel Radix Sort for Unbalanced Data
}

\author{
Martin Schmollinger \\ Wilhelm-Schickard-Institut für Informatik, Universität Tübingen \\ schmolli@informatik. uni-tuebingen. de
}

\begin{abstract}
Radix sort is an efficient method to sort integer keys on parallel computers. It is easy to parallelize and simple to implement. The main drawbacks of existing algorithms are load balancing problems and communication overhead. These problems are caused in data characteristics like data-skew and duplicates. There are several approaches how to parallelize the radix sort algorithm, which yield to reduce communication operations or to improve the load balance. If an algorithm has its focus on the optimization of the load balance then, its communication is inefficient. Otherwise, if the focus is on the communication minimization, then the algorithms are only efficient for well-distributed data. For the latter case, we will present an efficient improvement which helps to overcome the problems with unbalanced data characteristics. The suggested improvements are tested practically on a Linux-based SMP cluster.
\end{abstract}

\section{Introduction and Related Work}

Radix Sort is a method to sort a set of integer keys using their binary representation. The main idea is to sort the keys in several iterations. In each iteration the keys are sorted according to a certain number of bits (radix of size $r$ ), starting in the first iteration with the least significant bit. Assuming the keys consist of $l$ bits, the algorithm needs $l / r$ iterations to sort the keys. Detailed descriptions of sequential radix sort algorithms can be found in standard textbooks, see e.g. [13[10].

The main idea of the parallelization is that the processors are viewed as buckets. Roughly, each parallel iteration of radix sort consists of the following 3 steps. Each processor scans its local keys with a certain radix and stores them in the corresponding buckets. Then the sizes of the locally created buckets are exchanged by the processors in order to calculate a communication pattern for the buckets. The keys are exchanged according to the computed communication pattern. These steps are repeated until all bits of the keys were scanned. More detailed descriptions for shared-memory and distributedmemory machines can be found in [18].

The main problem with this approach is its irregularity in communication and computation which arise because of data characteristics like e.g. data skew or duplicates. Therefore, in [15] a load balanced parallel radix sort is presented. This algorithm splits the locally generated buckets with respect to balance the number of keys that have to be sent to each processor. Therefore, the resulting communication step is a real balanced all-to-all operation by the processors. 
Until now, all mentioned algorithms need $l / r$ key- and counter-communication steps. Especially for distributed-memory machines, where the communication is done over an interconnection network, this might be very time consuming. The communication and cache conscious radix sort algorithm (C3-Radix) [7] tries to improve this situation by starting the radix sort at the most significant bit. The intention of C3-Radix is to partition the data into several buckets which can be distributed equally among the processors. Therefore, the choice of the radix is very important. If the distribution of the buckets is not possible in a balanced way, the radix is enlarged and more fine-grained buckets are created. This is repeated until a good load-balance is achieved. Then the keys are sent with an all-to-all operation among the processors. The remaining task is to sort the buckets locally. For this step, the authors use a very efficient cache conscious radix sort algorithm which uses the fact that the data is already sorted for a certain number of bits. The algorithm has to communicate the keys only once, but because of the characteristics of the data there might be several iterations where the bucket counters have to be exchanged. Depending on the number of iterations and the size of the radix this can increase the running time of the algorithm extremely.

Another approach is based on Sample Sort which is often used in parallel sorting, see e.g. [63 2:14]. Each processor samples $q$ keys from its $N / P$ keys and exchanges them with the other processors. Sorting the set of samples in each processor makes it possible to create a set of $s-1<q$ equidistant keys called splitters. These splitters can be used to create $s$ buckets of approximately size $N / s$. Load balance can be achieved, if $s$ is a multiple of $P$. The parallel counting split radix sort algorithm (PCS-Radix) [9] uses Sample Sort for the partitioning of the data instead of using the radix of the keys directly. Radix sort is only used to sort the buckets locally. The prize for the independence of the data characteristics is the detection of global samples in the distributed system, but in a badly distributed environment this investment is worth doing. Despite of that, in environments where the data is mostly well distributed, the C3-Radix algorithm should be the algorithm of choice, because each processor is able to start the local creation of the buckets directly, and in general no further iterations are necessary to build the local buckets. On the other hand, also in a well-distributed environment, sometimes there may arise badly distributed data sets. The C3-Radix algorithm does not have the capability of being efficient in these cases, but it should be guaranteed that it is not far away from being efficient.

Hence, in the following we survey the possibilities for C3-Radix to be more stable and more predictable when working on unbalanced data distributions. In Section 2 , we sketch the C3-Radix without going into detail for the steps we will not improve and without describing their optimizations made in [7]. In Section 3, we will explain the problems of C3-Radix in cases of unbalanced data more deeply, we will give an example, and we will describe improvements. In Section 4 we will give and interpret results of experimental test for the improved algorithm, and in Section 5 we conclude.

\section{Outline of the C3-Radix Algorithm}

The algorithm sorts $N$ keys and each key consists of $l$ bits. The used radix has length $r$. Initially, each processor stores $N / P$ keys. The keys are globally sorted if the keys 
within each processor are sorted, and there is a known order of the processors for which all keys of one processor are greater oder equal to all keys in the preceding processor. Each processor builds buckets of keys by starting to observe the first $r$ bits of each key. The initial length of the radix should be chosen in the way that $2^{r}>P$. Keys with the same radix belong to the same bucket.

(1) Reverse Sorting. Each processor scans its $N / P$ integer keys using the first $r$ bits beginning with the most significant bit, and building the corresponding $2^{r}$ buckets. During the creation of the buckets a counter array is built, too. Each entry in the counter array contains the number of keys in the corresponding bucket. (2) Communication of Counters. The local $2^{r}$ counters are exchanged between the processors. After this step, each processor knows the total amount of elements per bucket. (3) Computation of bucket distribution. Each processor computes locally a distribution of the buckets to the processors. If it is not possible to achieve a good load balance, then each processor starts again with step 1 and sets the new radix to $i r$, where $i$ is the number of iterations. By extending the radix, the algorithm tries to produce more and smaller buckets that may lead to a better load balance. Otherwise the algorithm continues with step 4. (4) All-to-All key communication. The buckets are sent in an all-to-all fashion. After this step no more communication is necessary. (5) Local sorting.

\section{Improvements}

As we can see in Section 2, the main problem with the C3-Radix algorithm is that the first 3 steps may have to be repeated several times. The number of iterations depends on the data distribution, the initial radix chosen and the size of the integer keys. The more iterations are necessary, the bigger the radix gets. Since the number of buckets as well as the number of counters is $2^{r}$, the allocated memory and the amount of data, that has to be communicated may increase the running time of the algorithm tremendously. What we want to improve is the way, the algorithm tries to distribute the data equally among the processors if more than 1 iteration is necessary. All other optimizations of the C3-Radix algorithms will not be changed. In order to explain the problem more detailed, we give an example. Let $i$ be the number of iterations, then the accumulated number of counters in each processor is $\sum_{j=1}^{i} 2^{(j+1) r}$. In the experiments of [7] the radix is set to 5 . If we assume there is a data distribution that leads to 6 iterations, then the number of counters broadcasted over the network is $1,108,378,656$. If we further assume that a counter is a 32 bit integer (which is necessary for large $\mathrm{N}$ ), then we see that each processor has to communicate about 4.129 GB of data. Each processor sends its counter array to all the other $P-1$ processors. Assuming 16 processors, the total data transferred by the network is $990.96 \mathrm{~GB}$. Besides this communication problem, there might arise memory problems. At least each processor has to store the counter array and the buckets constructed locally. Although there are more data structures like for example the initial data (N/P elements) or different counter arrays for the communication operation, the memory demand is dominated by the counter array. Therefore, we take its estimated memory demand as a key figure. Since the counter array has size $2^{30}$ in the sixth iteration and each entry stores one 32 bit integer, each processor needs at least 4 GB of main memory. Depending on the implementation of the counter communication (allreduce, broadcast, ..) the node has 
to buffer up to $P$ counter arrays which leads to 64 GB in our example. For a huge part of supercomputers, namely the PC-based SMP- or workstation-clusters, this size is not manageable. The program will abort due to out of memory errors. In order to avoid these large data arrays, our idea is not to rebuild all the buckets and counters with a larger radix within each iteration, but only to rebuild those that lie directly on at least one border of two processor data domains. (The data domain of a processor is its part of the sorted sequence of $N$ integers. The $N / P$ smallest integers are the data domain of processor 0 , the next smallest $N / P$ integers are the data domain of processor 1, and so on). Hence, at most $P-1$ buckets need to be rebuilt.

In the first iteration the algorithm works as described in Section 2 . The only difference is that after checking if another iteration is necessary, only selected buckets are rebuild. For further iterations the first 3 steps are replaced by the following steps.

(1) Reverse Sorting of special buckets. Rebuild the buckets and counters that lie directly on the border of two processor data domains. (2) Communication of Counters. All-to-all communication of the new counter array. (3) Computation of bucket distribution. Check, if another iteration is necessary. If it is necessary, detect the buckets that have to be rebuilt with a higher radix. Otherwise proceed to step 4 of the algorithm described in Section 2

Now, we want to analyze the behavior of this new algorithm in order to judge the received improvement. In each iteration, a certain number of buckets have to be rebuild. We denote this number with $a_{j}$, where $1 \leq a_{j}<P$ and $j>0$ is the number of the iteration $\left(a_{1}=0\right)$. Let $i$ be the number of iterations performed. The accumulated amount of counters in each processor is $\sum_{j=1}^{i} 2^{r}+a_{j} 2^{r}-a_{j}=i 2^{r}+\left(2^{r}-1\right) \sum_{j=1}^{i} a_{j}$.

Looking at the scenario above $(r=5, i=6, P=16)$ and assuming the worst-case, where $a_{j}=P-1$ for $j>1$ the number of counters broadcasted over the network is 2517. Again we assume that a counter is a 32 bit integer, then we see that each processor has to communicate about $9.83 \mathrm{~KB}$. In each iteration each processor sends its counter array to the other $P-1$ processors. Hence, the overall amount of counters transferred by the network is $2359.2 \mathrm{~KB}$.

The situation is also much better for the memory requirements. Storing the counter array in step 6 requires $19.66 \mathrm{~KB}$ per processor which normally is negligible compared to custom computer memory sizes. It should fit in the cache! Again, the maximum temporary buffer size needed for the communication and reduction step of the counters is $314.56 \mathrm{~KB}$ which is not critical, too. The analysis and the example show the potential of the formulated improvement.

Until now, we have just looked how the number of counters increases in both methods depending on the number of iterations. But we also have to care about the way the new buckets are created locally in the step 1 (Reverse Sorting). C3-Radix scans its $N$ keys and rebuilds all buckets using the new radix. While building the buckets the counter array is updated, too. The scan can be done in $O(N)$, and an update of the buckets and counters can be done in $O(1)$. A practical drawback of this method is the following: As we know, the array of buckets and counters may get very big. Scanning the data means to access these arrays very irregularly. Hence, the memory hierarchy of the system is not 
used efficiently, and the time needed for step 1 will grow quickly beginning at a critical size of the radix.

On the other side, the improved version does not always have to scan all local integer keys. It just scans the keys located in the buckets, which were decided to be rebuild. But in the worst case all local keys are contained in such buckets. Further, these $N$ keys are not stored in one array consecutively, therefore, there is an additional overhead for switching between the buckets. After scanning the buckets and building new buckets out of them, these buckets have to replace the old buckets. Assuming that all buckets are stored in an array, this operation can be done in $O\left(2^{r}\right)$, whereby $r$ is the size of the initial radix in the first iteration. For small initial radix sizes $N$ should be $\geq 2^{r}$, hence, the additional time needed is not a problem. For larger sizes, step 1 is not the bottleneck of the algorithm as we saw in the example. Despite of that, we see that there might be situations for step 1, where C3-Radix is better than the improved version and vice versa, depending on the data distribution and the size of the radix.

In the following sections, we present experimental results that will show the behavior of the two algorithms for several data distributions. The improved algorithm is called balanced communication sensitive parallel radix sort (BCSP-Radix).

\section{Experimental Tests}

Our experimental tests were made on a Linux-SMP cluster with two Pentium III processors $(650 \mathrm{MHz})$ and $1 \mathrm{~GB}$ main memory per node. The nodes are connected by a Myrinet $1.28 \mathrm{GBit} / \mathrm{s}$ switched LAN. The whole cluster consists of 98 nodes (Kepler-Cluster, [16]). As we showed in Section 3, it is sufficient to compare the first 3 steps of C3and BCSP-Radix , because the steps 4 and 5 are the same as in the C3-Radix algorithm. We implemented them with $\mathrm{C}++$ and $\mathrm{TPO}++$ [4], which is an object-oriented message-passing system build on MPI [11,12]. Concerning the data distributions, we use two kinds of data sets. The first type are distributions already used in [15.7 9.5] and, therefore, are called standard data distributions. The second type are data distributions, which leads to worst-case behavior. We will explain them later on page 891, Duplicates are allowed in all data sets. For all experiments we try to achieve the best load balance possible. Since duplicates are allowed, a perfect load balance is not always possible. In general, we accept deviations of $\leq 1 \%$ of $N / P$.

Our four standard data distributions are defined as follows, in which MAX is $\left(2^{31}-1\right)$ for the integer keys, see also [157195]. Random [R], the data set is produced by calling the $\mathrm{C}$ library random number generator random () consecutively. The function returns integer values between 0 and $2^{31}-1$. Gaussian [G], an integer key is generated by calling the random ( ) function 4 times, adding the return values and dividing the result by 4 . Bucket Sorted [B], the generated integer keys are sorted into $P$ buckets, obtained by setting the first $N / P^{2}$ keys at each processor to be random numbers in the range of 0 to $(\operatorname{MAX} / P-1)$, the second $N / P^{2}$ keys in the range of $\operatorname{MAX} / P$ to $(2 \mathrm{MAX} / P-1)$, and so forth. Staggered [S], if the processor index $i$ is $<P / 2$, then all $N / P$ integer keys at the processor are random numbers between $(2 i+1) \operatorname{MAX} / P$. Otherwise, all $N / P$ keys are random numbers between $(i-P / 2) \operatorname{MAX} / P$ and $((i-P / 2+1) \operatorname{MAX} / P-1)$. 
The main problem of using radix sort is that we do not know how the data is distributed and, therefore, with which size of the radix we should start. Our aim is to minimize the total running time. But without knowing details about the data it is not possible to choose the size of the radix optimally. The algorithm should grant that the running time should be close or equal to its optimum independent of the radix size and data distribution. Hence, in our test, we varied the initial size of the radix from 5 to 12 . We made experiments using 16M integer keys and 8,16 and 32 processors. All tests produced similar results, therefore, we give the explanation of the results by means of the 16 processors test. The running times of this test are presented in Table 1

Table 1. Test with 16 processors ( 8 nodes with 2 processors) and $16 \mathrm{M}$ integer keys using 4 different data distributions, and varying the initial radix in a range from 5 to 12 .. The first number in each cell is the mean running time of all used processors for the first 3 steps. The second number is the number of iterations needed for the execution

\begin{tabular}{|c|c|c|c|c|c|c|c|c|c|}
\hline \multicolumn{10}{|c|}{ BCSP-Radix } \\
\hline radix & 5 & & 6 & 8 & 9 & 10 & 11 & 12 & total mean \\
\hline$[\mathrm{R}]$ & $0.95 \mid 1$ & $|1.06| 1$ & \begin{tabular}{l|l|l}
1 & $1.12 \mid 1$
\end{tabular} & $1.24 \mid 1$ & $1.24 \mid 1$ & $1.23|1|$ & $1.20 \mid 1$ & $|1.15| 1$ & 1.15 \\
\hline$[\mathrm{G}]$ & $2.63 \mid 3$ & $|2.42|^{2}$ & $2|1.85| 2$ & $1.68 \mid 2$ & $1.71 \mid 2$ & $1.77 \mid 2$ & $1.07 \mid 1$ & $1.11 \mid 1$ & 1.78 \\
\hline$[\mathrm{B}]$ & $2.87 \mid 3$ & $|2.87|^{3}$ & \begin{tabular}{l|l|l|}
3 & $2.51 \mid 2$ \\
\end{tabular} & $2.44 \mid 2$ & $2.10 \mid 2$ & $1.94 \mid 2$ & $0.93 \mid 1$ & 0.96 & 2.08 \\
\hline$[\mathrm{S}]$ & $2.76 \mid 3$ & $\left.2.53\right|^{3}$ & \begin{tabular}{|l|l|l|}
3 & $2.94 \mid 2$ \\
\end{tabular} & $2.34 \mid 2$ & $1.98 \mid 2$ & $1.79 \mid 2$ & $1.01 \mid 1$ & 1.09 & 2.05 \\
\hline \multicolumn{10}{|c|}{ C3-Radix } \\
\hline radix & 5 & & 6 & 8 & 9 & 10 & 11 & 12 & total mean \\
\hline$[\mathrm{R}]$ & $0.96 \mid 1$ & $|1.05| 1$ & \begin{tabular}{l|l|l|}
1 & $1.16 \mid 1$ \\
\end{tabular} & $1.23 \mid 1$ & $1.21 \mid 1$ & $1.19 \mid 1$ & $1.16 \mid 1$ & $1.14 \mid 1$ & 1.14 \\
\hline$[\mathrm{G}]$ & $2.87 \mid 3$ & $|1.63|^{2}$ & 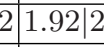 & $2.83 \mid 2$ & $\left.4.65\right|^{2}$ & \begin{tabular}{|l|}
$10.74 \mid 2$ \\
\end{tabular} & \begin{tabular}{|l|}
$1.06 \mid 1$ \\
\end{tabular} & $1.12 \mid 1$ & 3.35 \\
\hline$[\mathrm{B}]$ & $2.47 \mid 3$ & $4.453^{3}$ & $\begin{array}{ll}3 & 1.67 \mid 2 \\
\end{array}$ & $\left.2.09\right|^{2}$ & $3.74 \mid 2$ & $10.02 \mid 2$ & $0.93 \mid 1$ & 0.96 & 3.29 \\
\hline$[\mathrm{S}]$ & $2.49 \mid 3$ & $|4.23|^{3}$ & \begin{tabular}{l|l|l|}
3 & $1.75 \mid 2$ \\
\end{tabular} & $2.05 \mid 2$ & $3.62 \mid 2$ & 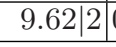 & $0.99 \mid 1$ & $1.08 \mid 1$ & 3.23 \\
\hline
\end{tabular}

Concerning the $[\mathrm{R}]$ distribution, both algorithms achieve similar running times for all sizes of the radix because, if only 1 iteration is necessary then they are the same. In this case, the best running time can be achieved by using the smallest radix, which is obvious, because then the data structures are small, too. Computation and communication benefit by that. In general, the overall best running time for all distributions can be achieved, if we use the smallest size of the radix, with which only 1 iteration is necessary. In our sample this is $r=11$ for [G], [B] and [S]. Again, both algorithms are the same. If the radix is chosen $<11$, then the situation is as follows. If the number of iterations needed to perform the algorithm is equal for several sizes of the initial radix, then C3Radix is better if the size of the radix is minimal and BCSP-Radix is better for larger sizes of the radix. The reason why C3-Radix gets slower for larger sizes of the radix is founded in the increasing sizes of the bucket and counter arrays. Communication and computation gets much higher if the radix size increases dramatically due to further iterations. BCSP-Radix is much more stable, because the more iterations are necessary, the smaller the buckets are that have to be rebuild. Furthermore, the size of the counter 
array for the communication is bounded by $O\left(2^{r}\right)$ as we saw in Section 3 . Therefore, the communication cost is stable, too. If BCSP-Radix is worse, this is because of the running time of step 1 . In these cases, due to the small radix, the data is distributed in a small number of buckets $(\leq P-1)$. Hence, each bucket has to be rebuild and each processor has to scan all integer keys stored locally. The additional time needed for replacing all buckets by $2^{r}$ new buckets leads to the worse behavior. An essential observation can be made looking at the mean of the processor mean times (see column total mean in Table 1]. BCSP-Radix is better for [S], [B], [G] and of course equal for [R]. While C3-Radix has some runaways in all distributions, BCSP-Radix is much more stable. This is important, because normally we do not know the behavior of the data with respect to the chosen radix. By using BCSP-Radix it is guaranteed that the total running time of the whole algorithm is not destroyed by the first 3 steps.

For the BCSP-Radix algorithm the worst-case data-distribution can be described as follows. After the first iteration, the data is partitioned into $P-1$ buckets of equal size and the other buckets are empty. The algorithm decides to rebuild all $P-1$ buckets with a larger radix. But the data is chosen in the way that the next bits are the same for all keys until the last $r$ bits begin. That means, that in each iteration the $P-1$ buckets remain unchanged until the last iteration. This is the worst case, because BCSP-Radix has to scan all keys in each iteration, and $P-1$ is the maximum number of buckets which achieve this situation. Using this data set with C3-Radix does also lead to the maximum number of iterations possible. This data set is not constructed very artificially. The keys are uniformly distributed within a small range of bits and duplicates are allowed. Hence, this might also occur in a well-distributed environment. As we know from the example in Section 3, we cannot perform all iterations for 32 bit keys with the C3-Radix, because the main memory of our SMP-nodes is limited to 1 GB (using only 1 processor per node!). Therefore, the data set is constructed in the way that the nodes of the cluster will not collapse ( $\leq 20$ bits). But we will show the behavior of BCSP-Radix performing all possible iterations.

Fig. 1 (left) presents an example of the comparison between the algorithms, where the data is distributed in the way that both algorithms have to perform 4 iterations, which means that the data can be partitioned looking at the first 20 bits. BCSP-Radix is much better than C3-Radix. The difference between both would even grow if further iterations were necessary. Unfortunately, C3-Radix cannot iterate further without aborting due to memory limitations.

In Fig.1 (right), the running times for BCSP-Radix making 6 iterations (30 bits) are illustrated. We have to adhere that in this case BCSP-Radix is even faster than C3-Radix performing only 4 iterations. Although this is a worst-case situation for BCSP-Radix, the running time is better than the worst time achieved with C3-Radix for [S], [B] and [G] and radix 10 where 2 iterations were necessary (see Table 1 ).

\section{Conclusions}

In the previous sections, we suggested improvements with respect to the data decomposition for the currently fastest parallel radix sort algorithm. Instead of rebuilding all buckets for each iteration with a larger radix size, BCSP-Radix only rebuilds those buck- 


\begin{tabular}{|c|c|c|c||c|}
\hline Algorithm & Step 1 & Step 2 & Step 3 & $\sum$ \\
\hline \hline C3 & 4.79 & 5.88 & 0.15 & 10.82 \\
\hline BCSP & 5.35 & 0.02 & $<0.01$ & 5.37 \\
\hline
\end{tabular}

\begin{tabular}{|c|c|c|c||c|}
\hline Algorithm & Step 1 & Step 2 & Step 3 & $\sum$ \\
\hline \hline BCSP & 7.72 & 0.04 & $<0.01$ & 7.76 \\
\hline
\end{tabular}

Fig. 1. Test with 16 processors, $16 \mathrm{M}$ integer keys, and an initial radix of 5 . The numbers are the mean times (in sec.) of the processors for a worst case situation. (left 4 iterations are necessary. (right) 6 iterations are necessary and only BCSP-Radix is able to terminate its execution

ets which help to find a better load balance for the proceeding steps of the algorithm. Due to this optimization, the computation, communication and memory requirements are much better. In experimental tests, we showed that the average general behavior of BCSP-Radix for standard data distributions is superior. While C3-Radix has several situations, where the execution time runs away, BCSP-Radix behaves much more stable and predictable. For worst-case data distributions the advantage of using BCSP-Radix is even bigger. While the non-optimized algorithm may not terminate due to memory constraints, BCSP-Radix is even in the position to achieve considerable running times. Even the memory requirements per processor are significantly within the capacity of custom workstations.

\section{References}

1. D. A. Bader and J. JáJá. SIMPLE: A Methodology for Programming High Performance Algorithms on Clusters of Symmetric Multiprocessors (SMPs). Journal of Parallel and Distributed Computing, 58(1):92-108, 1999.

2. G. E. Blelloch, C. E. Leiserson, B. M. Maggs, C. G. Plaxton, S. J. Smith, and M. Zagha. A Comparison of Sorting Algorithms for the Connection Machine. In Proceedings of Sysmposium on Parallel Algorithms and Architectures, pages 3-16, July 1991.

3. A. V. Gerbessiotis and C. J. Siniolakis. Deterministic Sorting and Randomized Median Finding on the BSP Model. In Proceedings oth the 8th ACM Symposium on Parallel Algorithms and Architectures, pages pp.223-232, 1996.

4. T. Grundmann, M. Ritt, and W. Rosenstiel. Object-Oriented Message-Passing with TPO++. In EURO-PAR 2000, Parallel Processing, volume 1900 of LNCS, pages 1081-1084. Springer Verlag, 2000.

5. D. R. Helman, D. A. Bader, and J. JáJá. Parallel Algorithms for Personalized Communication and Sorting With Experimental Study. In Proceedings of the IEEE Annual ACM Symposium on Parallel Algorithms and Architectures, pages 211-220, 1996.

6. D. R. Helman and J. JáJá. Sorting on Clusters of SMPs. Informatica: An International Journal of Computing and Informatics, 23, 1999.

7. D. Jiminez-Gonzales, J. Larriba-Pey, and J. Navarro. Communication Conscious Radix Sort. In Proceedings of the International Conference on Supercomputing, pages 76-82. ACM, 1999.

8. D. Jiminez-Gonzales, J. Larriba-Pey, and J. Navarro. GI-Seminar: Algorithms for Memory Hierarchies, volume 2625 of LNCS, chapter Case Study: Memory Conscious Parallel Sorting, pages 358-378. Springer Verlag, 2003. Advanced Lectures.

9. D. Jiminez-Gonzales, J. Navarro, , and J. Larriba-Pey. Fast Parallel In-Memory 64 Bit Sorting. In Proceedings of the International Conference on Supercomputing, pages 114-122. ACM, 2001. 
10. D. Knuth. The Art of Computer Programming: Sorting and Searching, volume 3. AddisonWesley, 1973.

11. Message Passing Interface Forum. MPI: A Message-Passing Interface Standard. Technical Report CS-94-230, Computer Science Department, University of Tennessee, Knoxville, TN, May 1994.

12. Message Passing Interface Forum. MPI-2: Extensions to the Message-Passing Interface, July 1997.

13. R. Sedgewick. Algorithms. Addison-Wesley, 1992.

14. H. Shi and J. Schaeffer. Parallel Sorting by Regular Sampling. Journal of Parallel and Distributed Computing, 14:361-372, 1992.

15. A. Sohn and Y. Kodama. Load Balanced Parallel Radix Sort. In Proceedings of the International Conference on Supercomputing, pages 305-312. ACM, 1998.

16. University of Tübingen (SFB-382). http://kepler.sfb382-zdv.uni-tuebingen.de/. 